\title{
Cadaveric Temporal Bone Dissection: Is It Obsolete Today?
}

\author{
Sulabha M. Naik ${ }^{1} \quad$ Mahendra S. Naik $^{1} \quad$ Nainjot Kaur Bains ${ }^{1}$ \\ ${ }^{1}$ Department of ENT, M.M. Institute of Medical Sciences and Research, \\ Mullana, Ambala, Haryana, India \\ Address for correspondence Dr. Sulabha M. Naik, MS, DORL, c/o Mr. \\ S. A. Naik, 9/4, Moti Mahal, 195, J. Tata Road, Churchgate, Mumbai \\ 400020, Maharashtra, India (e-mail: sulabha.naik@gmail.com).
} Int Arch Otorhinolaryngol 2014;18:63-67.

\begin{abstract}
Keywords

- cadaver

- temporal bone

- dissection

- simulation

Introduction Traditionally, surgical training in otology, is imparted by dissecting harvested human cadaveric temporal bones. However, maintenance of a cadaveric temporal bone laboratory is expensive and carries risk of exposure to infection. In recent times, other modalities of training are gaining ground and are likely to eventually replace cadaveric temporal bone dissection altogether.

Objectives Other alternative methods of training are emerging. New technology like simulation and virtual reality as high-fidelity, safer alternatives, are making rapid strides as teaching tools. Other options are the use of animal temporal bones as teaching tools. The advantages of these are compared.

Data Synthesis None of these modalities can replicate the innumerable anatomical variations which are a characteristic feature of the human temporal bone. A novice surgeon not only needs exposure to surgical anatomy and it's variations but also needs to develop hand-eye coordination skills to gain expertise.

Conclusion Deliberate practice on human cadaveric temporal bones only, will confer both mastery in anatomy and surgical technique. The human cadaveric temporal bone is ideal simulator for training in otology.
\end{abstract}

\section{Introduction}

Since the advent of the surgical microscope, otologic procedures have evolved into high-precision techniques. Methods of surgical training have also shown a technologically driven evolution. Use of temporal bones obtained from human cadavers has been an effective teaching tool. A recent training modality is by means of simulation. ${ }^{1}$ Synthetic replicas of temporal bones made of acrylic resin have been developed for trainee surgeons. ${ }^{2}$ These advanced training options will gradually replace traditional cadaveric temporal bone dissection and render it obsolete. We endorse the training of novice surgeons by use of cadaveric bones, both human and animal. The advantages and feasibility of this simple training technique are discussed. It remains a convenient teaching tool despite advances in technology.

received

January 4, 2013

accepted

March 10, 2013

\section{The Human Cadaveric Temporal Bone}

Cadavers and donated bodies remain an armament for teachers of human anatomy. ${ }^{3}$ Learning by "hands-on" training by dissection on human bodies is considered ideal. Acquiring the dexterity to drill the temporal bone, as well as mastering its anatomy, is the stepping-stone to successful ear surgery.

The temporal bone has a very complex configuration. It contains vital structures that are known for their anatomical variations. Different types of pneumatization of the mastoid air cell system, Korner's septum, anomalies of the facial nerve and ossicles, low-lying dura, anteriorly placed sigmoid sinus, and so on are likely to be encountered fairly often during mastoid and middle ear surgery. Dissection on cadaveric bones confers exposure to the myriad anomalies as well as confers the alertness to recognize an anomaly. Cawthorne ${ }^{4}$ has aptly stated that temporal bone surgery lends itself well to cadaveric practice, and before

Copyright $\odot 2014$ by Thieme Publicações License terms Ltda, Rio de Janeiro, Brazil

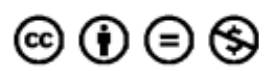


the undertaking of any new techniques, one should be conversant by means of cadaveric practice and also by observing experienced surgeons. He has also stated that facial nerve surgery needs experience and should be performed only after practice on cadaveric temporal bones. He opined that it is not possible to learn newer surgeries from written descriptions only, as the necessary details may not be available in an article. His observation remains strikingly relevant today, particularly with reference to facial nerve, stapes surgery, and cochlear implantation.

Dissection manuals, models, and simulation programs are inappropriate substitutes for the "hands-on" method of teaching human anatomy. George and De have emphasized that cadaveric temporal bone dissection also provides invaluable experience of anatomical variation. ${ }^{5}$ Anatomy is best mastered by serial dissection of human cadaveric temporal bones.

Resident training in otologic surgery in many centers includes dissection of preserved human temporal bones. The temporal bone dissection procedure involves harvesting the bone from a human cadaver and storage in formaldehyde. It is mounted securely in a bone holder in the same position as in actual surgery and dissected using a surgical microscope and a micromotor drill. This drilling may be compared with driving a car as it involves coordination of eye, hand, and foot and thus involves conditioning. George and De concurred that because temporal bone surgery is a challenging task, it is mandatory to develop the necessary motor skills along with simultaneous acquisition of a sound knowledge of temporal bone anatomy. ${ }^{5}$ They have reported that otologists with quality skills are the outcome of both cadaveric temporal bone dissection and surgical experience.

Fennessy and O'Sullivan stated the opinion that, because patient safety and surgeon competency are a concern, ${ }^{6}$ cadaveric temporal bone dissection is essential for surgical training. Strict legislation regarding organ donation in many nations has led to a shortage in the availability of temporal bones for surgical dissection. In the United Kingdom, a temporal bone laboratory has been established in Cork, which is the sole laboratory of its kind in Ireland. The resultant benefit to trainee otolaryngology residents in Cork is an improvement in surgical skills. ${ }^{6}$

Adoga et al at University of Jos, Nigeria conducted a study in which 18 bones in nine cadavers were dissected over a period of 3 months in preparation for cochlear implant and mastoid surgery. ${ }^{7}$ A total of 18 canal wall up, canal wall down mastoidectomies and cochlear implantation surgeries were performed on bones. They opined that use of fresh cadaver provides the closest ideal to human bones to demonstrate all anatomical features. Prior practice on cadavers will equip a novice with considerable manual dexterity. They concluded that temporal bone dissection confers a vital learning experience. Although this was a small study of 18 cadaveric bones, it nevertheless highlights the advantages of use of cadavers for surgical training.

In another study, the performance of otologists doing singular neurectomy (Gacek's operation) on cadavers was assessed by Feigl et al. ${ }^{8}$ They reported both an improved understanding of the anatomy and also improved ability to perform the procedure, following repeated practice of singular nerve neurectomy on a cadaver. This study also concluded that practice on cadavers leads to better results and is therefore needed for gaining experience.

An obvious disadvantage of the use of human cadaveric bones is the risk of transmission of infectious diseases, which is likely in the event of improper screening of donor cadavers. The bones are usually preserved in formaldehyde or frozen, but this carries a potential risk of transmission of hepatitis B. ${ }^{5}$ A serious hazard of cadaveric bones is the risk of prion transmission. ${ }^{9}$ The term prion means proteinaceous infectious organism. Prions can cause subacute encephalopathy namely Creutzfeldt-Jakob disease (CJD).There is a possibility of iatrogenic CJD via dura mater graft, and this is noteworthy, as dura mater is often accidently exposed during mastoid surgery as also during temporal bone dissection. Scott et al reported that the use of drill in mastoid surgery and temporal bone dissection leads to scattering of bone and soft tissue resulting in a residue, which they have termed bone dust. ${ }^{10}$ They have stated that neural tissue is present in this bone dust, and this is poses a hazard as the conjunctiva of the surgeon is exposed to aerosol inoculation with infected bone dust. To reduce the risk, precautions in the form of eye protection are mandatory both during surgery and cadaveric temporal bone dissection.

\section{Cadaveric Donation in India and UK}

In India, the Anatomy Act is a State Act, which is published in the State Government Gazette. ${ }^{11}$ The Anatomy Act of each state in India (e.g., Bombay Anatomy Act ${ }^{12}$ ) provides for the supply of unclaimed bodies to medical and teaching institutions for the purpose of anatomical dissection and other similar purposes. Cadavers used by these institutions are usually unclaimed bodies obtained by the police. There is a legal provision for obtaining unclaimed cadavers for purpose of dissection. ${ }^{3}$ In recent times cadavers are also being donated to teaching institutions, by relatives of the deceased, as per the wishes of the deceased.

All donors are screened for infectious diseases, such as AIDS. Infected cadavers are declined for donation, as it exposes all concerned personnel to the risk of contracting infections via this source. Various other factors exist (e.g., autopsied or decomposed body) that are likely to render a body unacceptable for an anatomical donation. The decision of rejection is to be made by the medical college at the time of donation. Under the law, every institution has the right to reject a body donation for any reason. ${ }^{3}$

Due to the huge demand for human cadavers for teaching, more efforts should be made to motivate the public toward body donation.

In the United Kingdom, the British anatomy Act of 1832 regulated the acquisition of cadavers for anatomical study. ${ }^{13}$ It is a universal practice in medical schools to include cadaveric dissection as part of anatomy teaching. ${ }^{5}$ In the undergraduate curriculum, however, dissection of the temporal bone is not included. As a result, at entry level, 
postgraduate students lack in-depth knowledge of the threedimensional anatomy of the temporal bone.

As per the 1984 and 1988 United Kingdom anatomy acts, the dissection of cadavers is to be confined solely for teaching of human anatomy. The use of cadavers for training of surgical technique is forbidden, ${ }^{5}$ despite the fact that cadaveric dissection of temporal bone is strongly recommended by the British Association of Otolaryngology and Head and Neck Surgeons. This legislation renders cadaveric dissection of temporal bone unviable in the United Kingdom.

In other nations, even if legislation permits, the cost of maintenance of the laboratories and the shortage of bones have become limiting factors.

\section{Surgical Training in Otology}

The conventional method of training of surgical residents begins with observing and assisting experienced surgeons. Present-day surgical microscopes are equipped with an observation tube or a camera connected to a display monitor. This facilitates step-by-step demonstration of the entire surgical procedure. After assisting an adequate number of cases, the trainees are permitted to perform surgery under assistance and guidance of a senior surgeon. Although this teaching protocol can be readily adopted for most procedures, it is not recommended for ear surgery. Common otologic procedures (e.g., mastoidectomy, cochlear implantation, labyrinthectomy, etc.) require drilling of the temporal bone for access to middle ear, mastoid, and inner ear. A high-speed drill equipped with a foot-operated switch is used for this purpose. The bone of the mastoid cortex is removed with the use of rotating burrs. The surgical microscope provides magnification, good illumination, and three-dimensional perception. The technique is meticulous and requires a great degree of precision. Extra caution needs to be exercised to avoid damage to lateral semicircular canal, facial nerve, tegmen plate, sinus plate, and so on.

\section{Simulation}

Wikipedia has defined simulation as "the imitation of the operation of a real-world process or system over time." ${ }^{14}$ An important application of simulation is for training in aviation and outer space, where high levels of reliability are needed. ${ }^{5}$ Simulation also is the basis of the computer gaming industry.

Due to difficulties faced in obtaining human temporal bones and the risks involved, new teaching tools are being developed. Wiet et al have performed pioneering research to develop a surgical simulator for temporal bone surgery. ${ }^{1}$ Imaging data combined with computer technology have been extrapolated to create simulation environments that mimic reality.

Okada et al constructed an artificial temporal bone by molding silicone and acrylic to devise a porous material, similar to the pneumatization of the mastoid. ${ }^{2}$ Vegetable parchment paper was used to create a tympanic membrane. They reported problems, such as tympanic membrane stiff- ness, greater density as compared with the real temporal bone, and the lack of a facial nerve. They concluded that while dissection in this otologic simulator does not replace actual dissection of temporal bones from cadavers, it offers a promising alternative for teaching.

Another anatomical replica of the temporal bone was devised by Bakhos et al. They subjected cadaveric temporal bones to helical computerized tomography, and the resultant data output was utilized to prepare a synthetic bone by means of stereolithography. ${ }^{15}$ They proposed that this model is good for surgical practice. They have extrapolated its utility as a tool for the planning of surgery of congenital middle ear anomalies, especially prosthesis insertion. Wiet et al have stated that to assimilate an in-depth knowledge of the temporal bone, dissection is required over 4 to 5 years. ${ }^{16}$ Plastic models will impart drilling practice, but the homogeneity of plastic is a serious limitation.

The VOXEL-MAN TempoSurg and Visible Ear Simulator are currently available commercially for middle ear and temporal bone surgery. ${ }^{17}$ Basically these are virtual reality simulators equipped with a haptic feedback. The TempoSurg simulator delivers a near-real experience and has utility as a tool for both novices and experienced surgeons especially while planning complex surgical procedures. Wiet et al have opined that these computer-generated simulators do not provide variance, which is characteristic of the temporal bone anatomy. ${ }^{16}$

However, in otology training, virtual reality and synthetic dissection models cannot be considered as a total replacement of the "live" environment on cadavers. Training on cadavers only provides the experience closest to real-time surgery. By virtual reality training, tactile, sensory, and stereognostic memory does not develop. ${ }^{11}$

George and De noted that they are in favor of the use of cadaveric temporal bones for the trainee otologist. ${ }^{5}$ However, they advocated the use of synthetic models and virtual simulators for junior trainees, owing to the fact that cadaveric bones are becoming increasingly scarce.

The use of animal ears is another viable training mode. Limited studies are available regarding animal ears, namely porcine and sheep. ${ }^{18,19}$ The temporal bone of the pig is an option as a learner's model but it has its limitations. Gurr et al conducted the first ever study of this type, which was not based on computer tomography. ${ }^{18}$ They claimed that the temporal bone of the pig differs from the human one, in that it is lacking in surgical landmarks, namely the temporal line and spine of Henle. The mastoid is also not pneumatized. The pig middle ear fortunately is similar to the human middle ear. They have suggested that it may be used for practicing bimanual motor skills necessary for microsurgery. They recommend it for learning middle procedures like myringoplasty and ossiculoplasty and grommet insertion, but not for mastoidectomy.

In stapes surgery, for technically difficult steps like stapedotomy, prior practice on cadaver bones is of great benefit. Gocer et al conducted a study based on sheep temporal bones, the cost of which is negligible. ${ }^{19}$ They have reported that 
sheep ossicles and facial nerve display a similarity to human ossicles and facial nerve. However, the sheep mastoid also lacks pneumatization. They recommended it as a training model for practice of ossiculoplasty, myringotomy, and particularly stapedectomy.

\section{Discussion}

Cadaveric temporal bone dissection courses are an available option for learners. Resident trainees currently gain exposure and expertise by attending cadaveric temporal bone dissection workshops, which are regularly conducted worldwide. ${ }^{5}$ Simulation techniques are favored currently in many countries because temporal bone laboratories are expensive to maintain and cadaveric specimens are difficult to obtain in sufficient quantity. Virtual reality temporal bone simulators offer a safe learning environment for developing familiarity with surgical anatomy along with a grasp of basic surgical skills. $^{20}$

In India, the shortage of human cadaveric temporal bones can be addressed. After a cadaver has been dissected in the anatomy department and prior to its disposal, the temporal bones should be harvested and preserved preferably in formaldehyde. A cadaveric temporal bone bank may be thus established. This will fulfill the requirement of cadaveric temporal bones for training in otology, otoneurology, and neurosurgery. Once temporal bones are harvested from cadavers of the anatomy department as a routine practice, then setting up a makeshift temporal bone dissection station is the next step. Fennessy and O'Sullivan have outlined the procedure for setting up a temporal bone laboratory. ${ }^{6}$ A temporal bone laboratory should have an operating microscope(a wall mounted model will suffice), a drill with burrs, instruments, a suction machine, a water connection, and facilities to store the bones. ${ }^{21}$ The use of animal bones for initial training, where human cadavers are scarce, is a practical option, but not without limitations. Animal bones are not exact replicas of human bones. Also, bones harvested from the pig may not be acceptable for religious reasons.

Surgical training of the future will certainly incorporate virtual simulation technology. It has been suggested that virtual simulation is a safe, customizable and accessible tool for acquisition of surgical exposure. ${ }^{20} \mathrm{~A}$ comparison of simulators of ear surgery has been done by de Sousa et al with the conclusion that they also play a significant role in training, but only as a complement. ${ }^{22}$ They also concurred that a simulator is far from ideal as a replacement for the human cadaveric bone, which itself is unique. Its duplication is not easy due to complex anatomical variations. Earlier, the high cost and lack of availability of this advanced technology made it difficult for it to be adopted as a standard teaching tool. However, the costs have now been reduced. ${ }^{5}$ Even so, in developing countries, surgical training using synthetic and animal models (real simulators) and virtual simulation techniques is a relatively new concept. Currently, there are no centers in India with access to this technology. This mode of training for novices will be feasible, only if the technology is made freely available and affordable.

\section{Conclusion}

New technology should not be viewed with skepticism and should be embraced wherever feasible. On the other hand, in the race for innovation and technology, we should not be swayed from reality. For the present time, it may be said that both virtual and real simulation-based training may be considered as an adjunct to traditional methods. Animal ears may also be employed for teaching difficult surgical steps. Virtual reality and synthetic replicas are a poor substitute for learning technique and decision making. Ergo, the human cadaveric temporal bone undoubtedly remains the best simulator for surgical training.

\section{Acknowledgments}

The Authors wish to thank Dr. V. V. Gopichand Patnaik, Principal, M.M. Institute of Medical Sciences and Research, Haryana, India and Ms. Balashri Jalgar, Associate, India Law Services, Mumbai, India for their valuable guidance in the preparation of this manuscript.

\section{References}

1 Wiet GJ, Rastatter JC, Bapna S, Packer M, Stredney D, Welling DB. Training otologic surgical skills through simulation-moving toward validation: a pilot study and lessons learned.J Grad Med Educ 2009;1:61-66

2 Okada DM, de Sousa AM, Huertas RdeA, Suzuki FA. Surgical simulator for temporal bone dissection training. Braz J Otorhinolaryngol 2010;76:575-578

3 Ajita R, Singh YI. Body donation and its relevance in anatomy learning: a review. J Anat Soc India 2007;56(1):44-47

4 Cawthorne T. The surgery of the temporal bone. Ann R Coll Surg Engl 1953;12:403-423

5 George AP, De R. Review of temporal bone dissection teaching: how it was, is and will be. J Laryngol Otol 2010;124:119-125

6 Fennessy BG, O'Sullivan P. Establishing a temporal bone laboratory: considerations for ENT specialist training. Ir J Med Sci 2009;178:393-395

7 Adoga SA, Maan ND, Umar BT, et al. Cadaver temporal bone dissection: the JOS experience. Webmed Central Otorhinolaryngology 2011;2:WMC001767. Available at: http://www.webmedcentral.com/article_view/1767. Accessed November 2, 2012

8 Feigl G, Kos I, Anderhuber F, Guyot JP, Fasel J. Development of surgical skill with singular neurectomy using human cadaveric temporal bones. Ann Anat 2008;190:316-323

9 Doerr HW, Cinatl J, Stürmer M, Rabenau HF. Prions and orthopedic surgery. Infection 2003;31:163-171

10 Scott A, De R, Sadek SAA, Garrido MC, Courteney-Harris RG. Temporal bone dissection: a possible route for prion transmission? J Laryngol Otol 2001;115:374-375

11 Patnaik VVG. Editorial. J Anat Soc India 2002;51(2):143-144

12 The Bombay Anatomy Act, 1949. Available at: http://bombayhighcourt.nic.in/libweb/acts/1949.11.pdf. Accessed November 2, 2012

13 British Anatomy Act, 1832. Available at: http://www.ancestryaid. co.uk/boards/family-history-genealogy-information/11515-anatomy-act-1832-a.html. Accessed July 19, 2013 
14 Wikipedia. Available at: http://en.wikipedia.org/wiki/Simulation. Accessed November 2, 2012

15 Bakhos D, Velut S, Robier A, Al zahrani M, Lescanne E. Threedimensional modeling of the temporal bone for surgical training. Otol Neurotol 2010;31:328-334

16 Wiet GJ, Stredney D, Wan D. Training and simulation in otolaryngology. Otolaryngol Clin North Am 2011;44:1333-1350, viii-ix

17 Clifton N, Klingmann C, Khalil H. Teaching otolaryngology skills through simulation. Eur Arch Otorhinolaryngol 2011;268:949-953

18 Gurr A, Kevenhörster K, Stark T, Pearson M, Dazert S. The common pig: a possible model for teaching ear surgery. Eur Arch Otorhinolaryngol 2010;267:213-217
19 Gocer C, Eryilmaz A, Genc U, Dagli M, Karabulut H, Iriz A. An alternative model for stapedectomy training in residency program: sheep cadaver ear. Eur Arch Otorhinolaryngol 2007;264: 1409-1412

20 Morris D, Sewell C, Barbagli F, Salisbury K, Blevins NH, Girod S. Visuohaptic simulation of bone surgery for training and evaluation. IEEE Comput Graph Appl 2006;26:48-57

21 Natarajan B, Baxter A. Preparation of a temporal bone exhibit. J Laryngol Otol 1994;108:9-12

22 de Sousa AMA, Okada DM, Suzuki FA. The use of simulators in the learning for otologic surgery. Int Arch Otorhinolaryngol 2011;15 (4):509-514 